$$
\begin{gathered}
\text { RECEIVED } \\
\text { AUG } 24 \text { G998-A22876 } \\
\text { OS TI } \\
\text { CONF- } 980560 \ldots
\end{gathered}
$$

\title{
QUANTITATIVE COMPARISONS BETWEEN EXPERIMENTALLY MEASURED 2-D CARBON RADIATION AND MONTE CARLO IMPURITY (MCl) CODE SIMULATIONS
}

\author{
by \\ T.E. EVANS, D.F. FINKENTHAL, M.E. FENSTERMACHER, \\ A.W. LEONARD, G.D. PORTER, and W.P. WEST
}

DISTABUTION OF THS DOCUMENT IS UNLIMTED $h$ MASTER

AUGUST 1998 


\section{DISCLAIMER}

This report was prepared as an account of work sponsored by an agency of the United States Government. Neither the United States Government nor any agency thereof, nor any of their employees, makes any warranty, express or implied, or assumes any legal liability or responsibility for the accuracy, completeness, or usefulness of any information, apparatus, product, or process disclosed, or represents that its use would not infringe privately owned rights. Reference herein to any specific commercial product, process, or service by trade name, trademark, manufacturer, or otherwise, does not necessarily constitute or imply its endorsement, recommendation, or favoring by the United States Government or any agency thereof. The views and opinions of authors expressed herein do not necessarily state or reflect those of the United States Government or any agency thereof. 


\section{DISCLAIMER}

Portions of this document may be illegible electronic image products. Images are produced from the best available original document. 


\title{
QUANTITATIVE COMPARISONS BETWEEN EXPERIMENTALLY MEASURED 2-D CARBON RADIATION AND MONTE CARLO IMPURITY (MCl) CODE SIMULATIONS
}

by

T.E. EVANS, D.F. FINKENTHAL, † M.E. FENSTERMACHER, $\ddagger$ A.W. LEONARD, G.D. PORTER, $\neq$ and W.P. WEST

This is a preprint of a paper to be presented at the 13th International Conference on Plasma Surface Interactions in Controlled Fusion Devices, May 18-23, 1998, San Diego, California and to be published in Journal of Nuclear Materials.

tPalomar College

FLawrence Livermore National Laboratory

\author{
Work supported by \\ the U.S. Department of Energy \\ under Contracts DE-AC03-89ER51114 and W-7405-ENG-48
}

GA PROJECT 3466

AUGUST 1998 


\section{ABSTRACT}

Experimentally measured carbon line emissions and total radiated power distributions from the DIII-D divertor and Scrape-Off Layer (SOL) are compared to those calculated with the Monte Carlo Impurity (MCI) model. A UEDGE [1] background plasma is used in MCI with the Roth and Garcia-Rosales (RG-R) chemical sputtering model [2] and/or one of six physical sputtering models. While results from these simulations do not reproduce all of the features seen in the experimentally measured radiation patterns, the total radiated power calculated in MCI is in relatively good agreement with that measured by the DIII-D bolometric system when the Smith78 [12] physical sputtering model is coupled to RG-R chemical sputtering in an unaltered UEDGE plasma. Alternatively, MCI simulations done with UEDGE background ion temperatures along the divertor target plates adjusted to better match those measured in the experiment resulted in three physical sputtering models which when coupled to the RG-R model gave a total radiated power that was within $10 \%$ of measured value. 
2 


\section{INTRODUCTION}

The DIII-D Monte Carlo Impurity (MCI) code uses a linear quasi-kinetic approach (i.e. it follows individual particles but does not include the full range of drift kinetic effects as is done in a Fokker-Planck code) to model the generation and transport of carbon particles [3]. This approach is particularly well suited for studying the atomic and molecular physics of carbon impurities near plasma facing material surfaces and in regions where the background plasma parameter gradient scale lengths are small compared to an impurity ion, neutral or molecular mean free path length. MCI typically uses a deuterium background plasma $\left(\mathrm{D}^{+}\right)$ solution generated by the UEDGE fluid code [1] along with several types of physical sputtering models coupled to the Roth and Garcia-Rosales (RG-R) chemical sputtering model [2]. Deuterated methane $\left(\mathrm{CD}_{4}\right)$ yields from the $\mathrm{RG}-\mathrm{R}$ chemical sputtering model and/or atomic neutral carbon yields from one of the physical sputtering models are calculated on each segment of the DIII-D walls and divertor target plates. The sputtered particles are launched from each surface segment with a 3-D cosine distribution and a velocity based on the incident energy of the sputtering particle.

MCI simulations follow each sputtered carbon particle from birth to death across the computational domain which covers the region from the $98 \%$ flux surface to the vessel walls and divertor target plates. A particle tracking algorithm displays the current charge state of the impurity at each time step. This allows us to assess the impact of various forces acting on the impurities in the divertor and SOL and to determine how local transport parameters such as background ion flows and temperature gradients affect the impurity density distributions and radiation rates. In addition, ensembles of tracks originating from various plasma facing surface provide us with valuable statistics on core penetration and surface redeposition probabilities which are compared with experimental measurements.

One of the primary goals of the MCI project is to develop a good understanding of the carbon sputtering physics needed to reliably model DIII-D plasmas. As seen from Fig. 1, physical sputtering yields from carbon surfaces are highly nonlinear. A factor of two change in the energy of the incident deuterium and carbon flux can result in an order of magnitude increase in the sputtering yield. In addition, the self-sputtering process can potentially lead to an explosive growth in the carbon production rate which may cause substantial changes in the background plasma. Under some conditions a strong self-sputtering amplification may occur depending on the local plasma parameters just in front of the sputtering site. Thus, in a selfconsistent model, it is necessary to understand the response of the plasma to the carbon radiation and how this influences the properties of the particle flux impinging on the target plates. While a self-consistent nonlinear model for this process does not yet exist, a first step 
in this direction is to determine which of the existing physical sputtering models best agrees with data taken from high power tokamak plasmas and if chemical sputtering as prescribed by the RG-R model is required to match the experimental data.

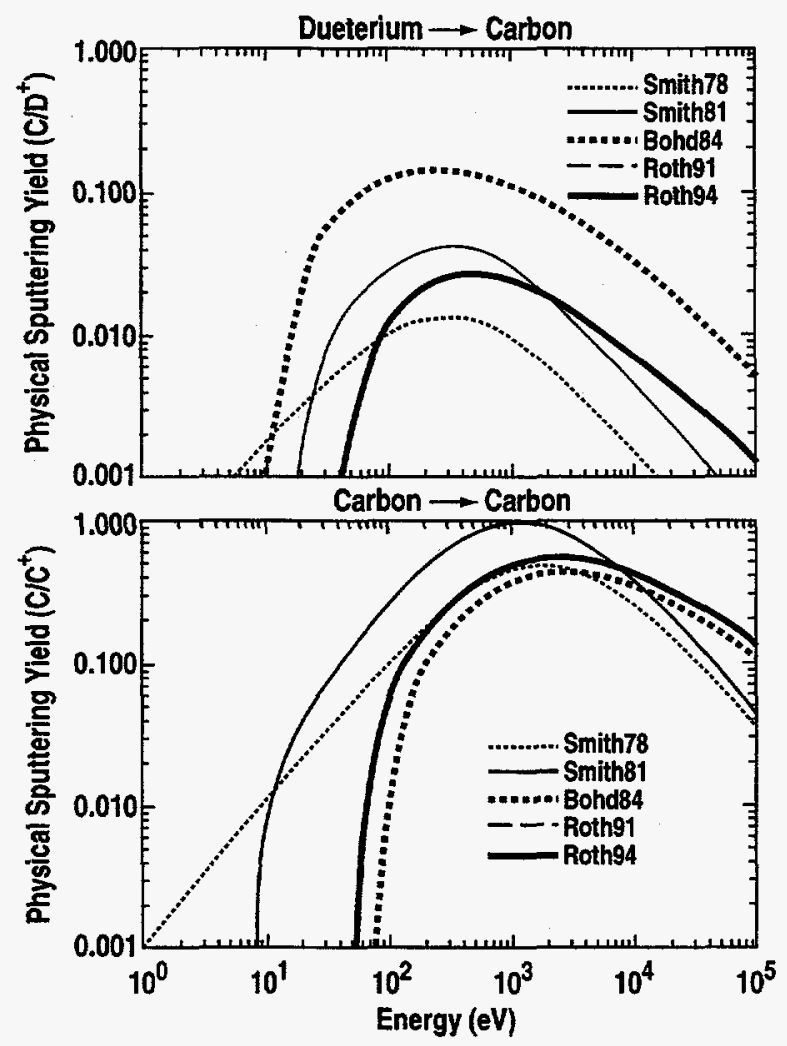

Fig. 1. Atomic carbon yields for an incident $D^{+}$(above) and carbon ion (below) as a function of the energy of the incident particle for each of the physical sputtering models used in $M C I$ except $Y_{p}=$ const. 


\section{MCI RADIATION BENCHMARKING BACKGROUND}

One of the most rigorous tests of a divertor simulation code is a qualitative and quantitative comparison of simulated 2-D radiation distributions with those measured experimentally. This comparison is being done in $\mathrm{MCI}$ for both individual carbon emission lines and the total radiated power from the divertor and SOL. For this, 2-D spatial images of $\lambda=465 \mathrm{~nm}$ the $\mathrm{C}^{2+}$ line emissions, are obtained from an optically filtered Tangential TV (TTV) system viewing the divertor X-point region [4]. Since the TTV system is not absolutely calibrated, only the qualitative features of the poloidal distribution i.e., the locations of bright emission spots can be compared to those calculated in MCI. Good qualitative agreements are found for some plasmas conditions [5] but significant differences can also exist. In addition, absolutely calibrated data from the DIII-D bolometer system [6] is inverted on a $65 \times 65$ poloidal grid to create 2-D images of the total radiated power. MCI calculations of the total power radiated by carbon are then compared qualitatively and quantitatively to these bolometer images. Additionally, the bolometer and MCI data are spatially integrated over the divertor and SOL in order to make quantitative comparisons of the total power radiated from the open field region of the plasma.

The basic impurity transport models used in MCI are intrinsically 3-D but typically rely on an axisymmetric background plasma solution from the UEDGE code. MCI calculates charge dependent carbon density distributions in a toroidal space which accurately represents the geometry of the DIII-D walls, divertor target plates, flux surfaces and edge plasma. Carbon density distributions are projected on to a 2-D (R,z) grid which represents the poloidal cross section of the DIII-D tokamak. Carbon line emissivities are calculated and displayed on the same 2-D grid for comparisons with inverted data from the TTV system. The total radiated power calculated from ADPAK [7] data in MCI, is also displayed on the same poloidal grid for comparisons with the bolometer images.

One of six normal incidence physical sputtering models is used in MCI to specify the atomic carbon yield $\mathrm{Y}_{\mathrm{p}}$. Each of these physical sputtering models may also be coupled to the RG-R model. In this case the total sputtering yield is $Y_{T}=Y_{p}+Y_{c}$ where $Y_{c}$ is the $C D_{4}$ yield calculated with the RG-R model. In the RG-R model $Y_{c}$ is comprised of a surface term $Y_{s}$ and a thermal term $Y_{t}$ such that $Y_{c}=Y_{s}+Y_{t}\left(1+B Y_{p}\right)$. Here $B$ is a fitting parameter which depends on the mass of the hydrogen isotope striking the carbon surface. For deuterium $B$ is set to 125 [2]. The dependencies of $Y_{S}$ and $Y_{t}$ on the surface temperature, incident particle energy, and particle flux are given in ref. 2 where a revised Bohdansky [8] physical sputtering model was used to calculate $Y_{p}$. We refer to the Bohdansky model as Bohd84 and the revised Bohd84 model used in Ref. 2 as the Roth94 model [9]. MCI also has the option of using an 
earlier version of the Roth94 model. We refer to this option as the Roth91 model [10]. Each of these physical sputtering models is derived from a set of relatively well known physical processes governing the interaction of particles with solid surfaces [11]. They have been benchmarked against ion beam sputtering data but have yet to be rigorously tested against tokamak data. The processes involved in chemical sputtering are not as well established. A second class of physical sputtering models are those based on experimental data from high energy, low flux, ion beams impinging on smooth solid surfaces. MCI currently uses two such semi-empirical models. One is referred to as the Smith78 model [12] and the other is the Smith81 model [13]. Smith81 includes a specific sputtering threshold dependence on the energy of incident particles and a somewhat different scaling with the mass and atomic number of the incident particle than Smith78. The final physical sputtering option used in MCI is $\mathrm{Y}_{\mathrm{p}}=$ constant. This option is used primarily for comparisons with atomic carbon sources calculated in UEDGE [14].

A comparison of atomic carbon yields for each of $\mathrm{MCI}$ 's physical sputtering models except $Y_{p}=$ const., is shown in Fig. 1. During a typical MCI simulation, deuterium and impurity ions are accelerated by the electrostatic sheath which forms in front of the carbon surface. The sheath potential is calculated in $\mathrm{MCI}$ and introduces a sensitive $\mathrm{T}_{\mathrm{e}}$ dependence into the physical sputtering models due to changes in the ion acceleration across the sheath layer. MCI has switches for modeling the incident flux either as mono-energetic particles or with an analytically specified energy distribution function such as a Maxwellian. In addition, the $\mathrm{D}^{+}$energy at the plasma-sheath boundary may be taken either directly from the UEDGE background or derived from divertor Langmuir probe and divertor Thomson data. Each carbon ion's charge state and energy is known at the plasma-sheath boundary from the MCI simulations. ELMs and non-Maxwellian tails are also expected to have a significant impact on $\mathrm{Y}_{\mathrm{p}}$ but as yet are not explicitly modeled in $\mathrm{MCI}$.

The simulations discussed here are for DIII-D shot 87506 at $t=2240 \mathrm{~ms}$. At this time, the plasma was in an ELMing $\mathrm{H}$-mode phase with an attached outer strike point and a detached inner strike point. The injected neutral beam power was $4900 \mathrm{~kW}$ and the total radiated power, as measured by the bolometer system, was $2182 \mathrm{~kW}$. The divertor and SOL radiation accounts for $1718 \mathrm{~kW}$ of this while the remainder comes from the core plasma. The integrated inner divertor heat flux, measured by the IRTV camera, was $364 \mathrm{~kW}$ and the outer divertor heat flux was $1419 \mathrm{~kW}$. Approximately $20 \%$ of the input power was unaccounted for at this time in the shot. A UEDGE background solution was generated and matched to a range of experimental measurements taken from this plasma. This matching process involves obtaining the best agreement possible with each of the available diagnostics. Nevertheless, significant differences between some of the UEDGE plasma parameters and the experimental measurements can not always be avoided. 
For the case considered here, UEDGE found peaks in $\mathrm{T}_{\mathrm{i}}$ of $1.92 \mathrm{eV}$ and $8.09 \mathrm{eV}$ near the inner and outer strike points, respectively. These corresponded to a peak in the $\mathrm{D}^{+}$flux near the outer strike point of approximately $3.1 \times 10^{23}$ particles $/ \mathrm{m}^{2} / \mathrm{s}$ and one near the inner strike point of about $1.7 \times 10^{23}$ particles $/ \mathrm{m}^{2} / \mathrm{s}$. Using these parameters and $\mathrm{T}_{\mathrm{e}}$ from UEDGE, MCI found incident $\mathrm{D}^{+}$energies of 10 and $44 \mathrm{eV}$ at the inner and outer strike points peaks, respectively. Experimental $T_{e}$ measurements made in the outer SOL, suggest that UEDGE's $T_{i}$ values are about a factor of 2-3 low for this shot. The divertor Langmuir probe located at $R$ $=1.48 \mathrm{~m}$, found a $\mathrm{T}_{\mathrm{e}}=17 \pm 2.5 \mathrm{eV}$ while the divertor Thomson system, measuring a point just above the surface of the target plate, found $\mathrm{T}_{e}=15 \pm 5 \mathrm{eV}$ at this radius. At this time in the shot, the outer strike point position was $R=1.45 \mathrm{~m}$. Thus, based on the experimental measurements we expect a peak temperature near the outer strike point of 30 to $40 \mathrm{eV}$, assuming an e-folding length of about $0.03 \mathrm{~m}$. It is also noted that the UEDGE solution had a rather flat radial temperature profile which is not typically observed in similar shots with strike point sweeping. To allow for these differences MCI simulations were done using both the UEDGE solution along the target plates and UEDGE data scaled by a factor of 2.5 to better match the experimental measurements. We also note that the experimental measurements reveal temperature fluctuations ranging between 15 and $35 \mathrm{eV}$ with periodic spikes up to $80 \mathrm{eV}$ during an ELM. 
6 


\section{RESULTS AND DISCUSSION}

First, we discuss results from qualitative comparisons between the measured $\mathrm{R}, \mathrm{z} \mathrm{C}^{2+}$ line emission and those simulated in MCI. The upper portion of Fig. 2 shows the experimental $\mathrm{C}^{2+}$ line emission at $\mathrm{t}=2240 \mathrm{~ms}$ for shot 87506 . The brightest point in this images is located about $0.03 \mathrm{~m}$ above the target plate near the outer strike point. An extended zone of radiation also spans the region from the outer strike point, along the outer leg, up to the $\mathrm{X}$-point where it connects to a second band of relatively high emission centered in the SOL just inside the $\mathrm{X}$-point region. Results from an MCI simulation, done on a standard UEDGE grid with the Roth94 physical sputtering model coupled to the RG-R chemical process, are shown in the lower portion of Fig. 2. MCI reproduces some of the qualitative features of the $\mathrm{C}^{2+}$ line emission along the outer leg but significantly underestimates the SOL radiation just inside the $\mathrm{X}$-point. $\mathrm{MCI}$ also indicates a band of intense $\mathrm{C}^{2+}$ line emission along the surface of the outer target plates extending outward from the strike point into the SOL. This region, which has a radial extend of $0.05 \mathrm{~m}$ includes zones of radiation with intensities which range between $130 \%-160 \%$ of that in the peak near the outer divertor leg. The TTV data indicates low radiation intensities in this region suggesting that the $\mathrm{MCI}$ has overestimated the $\mathrm{C}^{2+}$ line emission along the outer target plates. The background plasma $\mathrm{T}_{\mathrm{e}}$ just inside the $\mathrm{X}$-point is of order $40-75 \mathrm{eV}$. When this temperature is artificially reduced in the $\mathrm{MCI}$ simulations the $\mathrm{C}^{2+}$ emission from that region increases. This indicates that UEDGE solution is not accurate enough near the $\mathrm{X}$-point.

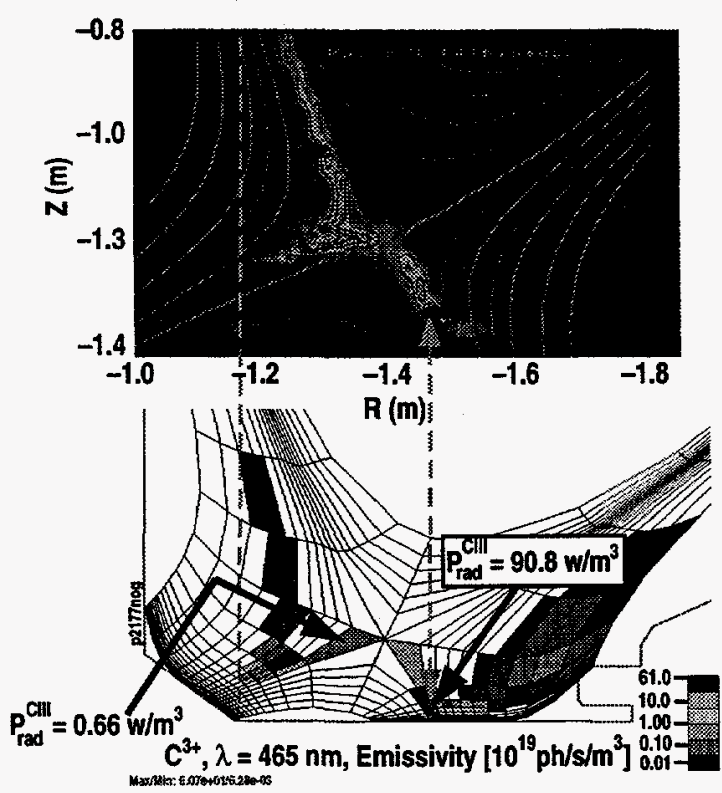

Fig. 2. Upper - experimentally measured $C^{2+}, \lambda=465 \mathrm{~nm}$, line emission from the TTV system. Lower calculated $C^{2+}, \lambda=465 \mathrm{~nm}$, line emission from an MCI simulation where the Roth94 physical sputtering model is coupled to the RG-R chemical sputtering model. 
Next we look at the 2-D distributions of the total radiated power in the divertor. Figure 3 shows a poloidal image of the total radiated power measured by the bolometer (upper) as compared to the total power radiated by carbon from the MCI simulation (lower). The MCI results represent a sum over all the spectral lines in each ionization state of carbon for the same conditions as in Fig. 2. Experimentally, the primary source of radiation is located in the SOL near the region just inside the $\mathrm{X}$-point and to a lesser extent from a small zone located in the outer SOL between the divertor baffle plate and the outer leg about $0.08 \mathrm{~m}$ above the divertor floor. The large radiation zone in the SOL just inside the $\mathrm{X}$-point is responsible for approximately $25 \%$ of the radiated power from the divertor while the smaller zone in the outer SOL contributes about $350 \mathrm{~kW}$ to the integrated divertor radiation. MCI has an intense band of radiation centered along the outer leg of the divertor which contributes about $440 \mathrm{~kW}$ or approximately $45 \%$ of the integrated power radiated by carbon from the divertor. The total divertor and SOL radiation calculated in MCI is $998 \mathrm{~kW}$ while that measured with the bolometer is $1718 \mathrm{~kW}$.

Figure 1 suggests that it mãy be possible to obtain better agreement in the radiation by coupling one of the other physical sputtering models to the RG-R chemical sputtering process. A series of $12 \mathrm{MCI}$ simulations were run to test this hypothesis. Table 1 provides a summary of the carbon source rate summed over all the sputtering sites, the resulting carbon inventory integrated over the entire computational domain and each ionization state, and the total power radiated from carbon in the divertor and SOL plasma for each of the 12 cases. The rows in the table represent results from each of $\mathrm{MCI}$ 's physical sputtering models. All of the data in Table 1 were produced using the unaltered UEDGE background solution on the UEDGE grid. As seen from this table, the MCI solution with Smith78 coupled to the RG-R process provides a relatively good match to the experimentally measured radiation.

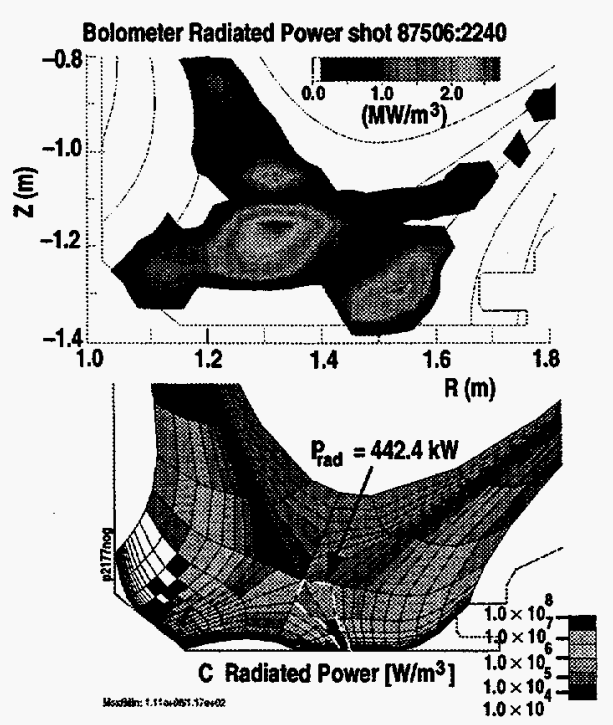

Fig. 3. Upper - experimentally measured total radiation from the DIII-D bolometer system. Lower - total radiation from carbon calculated by MCI for the same conditions as in Fig. 2. 
Table 1

Comparison of total carbon source rate, inventory and radiated power for each of the $\mathrm{MCl}$

physical sputtering models with and without chemical sputtering from the RG-R model. The unaltered UEDGE plasma solution was used along the target plates to calculate the carbon sputtering yield

\begin{tabular}{llrrr}
\hline \multicolumn{1}{c}{$\mathrm{Y}_{\mathrm{p}}$ model } & \multicolumn{1}{c}{$\mathrm{Y}_{\mathrm{c}}$ model } & $\begin{array}{c}\text { C source rate } \\
(\mathrm{A})\end{array}$ & $\begin{array}{c}\text { C inventory } \\
(\mathrm{x} \mathrm{10})\end{array}$ & $\begin{array}{c}\text { Rad. Power } \\
(\mathrm{kW})\end{array}$ \\
\hline Roth94 & RG-R & 219.8 & 9.336 & 998 \\
Smith78 & RG-R & 379.2 & 14.930 & 1626 \\
Smith81 & RG-R & 517.5 & 12.140 & 1489 \\
Bohd84 & RG-R & 2150.0 & 27.510 & 4110 \\
Roth91 & RG-R & 211.5 & 9.460 & 1001 \\
Y $_{\mathrm{p}}=10^{-3}$ & RG-R & 235.6 & 15.190 & 1516 \\
Roth94 & none & 19.5 & 0.374 & 49 \\
Smith78 & none & 121.6 & 3.978 & 460 \\
Smith81 & none & 199.2 & 2.199 & 356 \\
Bohd84 & none & 1205.1 & 12.260 & 1994 \\
Roth91 & none & 14.6 & 0.305 & 41 \\
Y $=10-3$ & none & 31.6 & 2.533 & 258 \\
\hline
\end{tabular}

Slight changes were observed in the simulated 2-D distributions for each of these cases but, generally speaking, the qualitative 2-D comparisons between distributions simulated in MCI and those measured with the TTV or bolometer systems had significant mismatches along the divertor target plates and in the SOL just inside the $\mathrm{X}$-point.

Since physical sputtering yields are a strong function of incident energy, and the UEDGE solution used in the simulations above have target plate temperatures which are significantly less than those measured experimentally, it is of interest to adjust the UEDGE solution accordingly. Thus, a second series of MCI simulations were done in which $T_{e}$ and $T_{i}$ were increased by a factor of 2.5 in each cell along the target plates compared to the original UEDGE value. It is noted that a change in $T_{i}$ from $10 \mathrm{eV}$ to $25 \mathrm{eV}$ increases the incident energy of the $\mathrm{D}^{+}$ions at the target plate surface to approximately $110 \mathrm{eV}$. At $110 \mathrm{eV} \mathrm{D} \mathrm{D}^{+}$ions produce physical sputtering yields relatively close to each of the peaks shown in Fig. 1(upper). Results from MCI simulations using the adjusted UEDGE target plate plasma temperatures are summarized in Table 2. Comparing these results to those obtained with the unaltered UEDGE target plate plasmas, we see a substantial increase in the radiated power, carbon inventory, and carbon source rate for each case except those with $Y_{p}=10^{-3}$. In the $Y_{p}$ $=10^{-3}$ case, a modest increase in the radiated power results from a somewhat enhanced 
Table 2

The same as Table 1 except the UEDGE solution along the target plates is adjusted to better match the experimental data

\begin{tabular}{llrcc}
\hline \multicolumn{1}{c}{$\mathrm{Y}_{\mathrm{p}}$ model } & \multicolumn{1}{c}{$\mathrm{Y}_{\mathrm{c}}$ model } & $\begin{array}{c}\text { C source rate } \\
(\mathrm{A})\end{array}$ & $\begin{array}{c}\text { C inventory } \\
(\mathrm{x} \mathrm{10})\end{array}$ & $\begin{array}{c}\text { Rad. Power } \\
(\mathrm{kW})\end{array}$ \\
\hline Roth94 & RG-R & 496.3 & 15.270 & 1690 \\
Smith78 & RG-R & 533.5 & 27.880 & 2862 \\
Smith81 & RG-R & 1078.4 & 30.470 & 3467 \\
Bohd84 & RG-R & 4292.1 & 125.700 & 14190 \\
Roth91 & RG-R & 478.4 & 16.630 & 1721 \\
Yp $10^{-3}$ & RG-R & 225.1 & 16.000 & 1573 \\
Roth94 & none & 192.0 & 2.633 & 384 \\
Smith78 & none & 229.1 & 9.822 & 1096 \\
Smith81 & none & 566.5 & 10.690 & 1344 \\
Bohd84 & none & 2637.9 & 87.510 & 9961 \\
Roth91 & none & 180.8 & 2.353 & 346 \\
Y $=10^{-3}$ & none & 31.6 & 2.768 & 272 \\
\hline
\end{tabular}

atomic neutral penetration probability due to the launch energy dependence on the incident particles.

As seen in Table 2, three of MCI's sputtering options fall within $10 \%$ of the radiated power measured by the bolometer. These are the Roth94 + RG-R, the Roth91 + RG-R, and $Y_{p}=10^{-3}+R G-R$. On the other hand, measurements of CD band emissions near the DIII-D target plates are particularly weak compared the $C^{1+}$ line emissions [15] suggesting a relatively modest amount of chemical sputtering compared to the physical sputtering level. Considering the uncertainties involved in the UEDGE solution and the sputtering models, i.e., incidence angle dependencies, surface roughness, dust and coating effects, etc., as well as uncertainties in the experimental data and those associated with temperature fluctuations and nonlinear transport effects, it is also plausible that either the Smith78 or the Smith81 model alone may ultimately account for all of the radiated power observed with the bolometer. 


\section{SUMMARY AND CONCLUSIONS}

Given the inherent uncertainties in the UEDGE and MCI models along with those in the experimental data, it is not yet possible to estimate the accuracy of the RG-R chemical sputtering process or to establish which physical sputtering model best accounts for the experimental measurements. In ELMing $\mathrm{H}$-mode plasmas, with an attached outer strike point and a detached inner strike point, there are substantial uncertainities associated with the dynamics and toroidal/radial distributions of heat and particle fluxes driven by the ELMs. These can have a significant impact on the sputtering models as well as nonlinear effects associated with atomic processes which are responsible for establishing the radiation rates in the divertor.

By better matching the UEDGE ion temperatures to experimentally measured values along the divertor target plates, three of MCI's sputtering options resulted in total radiated powers which were within $10 \%$ of those measured experimentally. In each of the cases studied, the 2-D radiation distributions calculated in MCI do not generally match those measured in the experiment . In addition, large variations in the total radiated power with different sputtering options have relatively little impact on the 2-D distributions suggesting that the mismatch arises primarily in the background plasma.

MCI simulations emphasize the importance of accurately matching the background plasma solution with each of the available experimental measurements. Without well matched background plasma solutions uncertainties in the sputtering and impurity transport physics can not be resolved in the MCI model. Significant improvements in the both fluid and Monte Carlo code are needed before these issues can be satisfactorily resolved. Finally, a two way coupling of the fluid and Monte Carlo codes needs to be implemented in order to assess the full impact of nonliearities in the sputtering on the background plasma solutions. 


\section{REFERENCES}

[1] T.D. Rognlien, et al., J. Nucl. Mater. 196-198 (1992) 347.

[2] J. Roth and C. García-Rosales, Nucl. Fusion 36 (1996) 1647.

[3] T.E. Evans and D.F. Finkenthal, to appear in Atomic Processes in Plasmas, Ed., American Institute of Physics Conf. Proc., Vol. , AIP Press, New York, 1998.

[4] M.E. Fenstermacher,et al., Rev. Sci. Instru. 68 (1997) 974.

[5] S.L. Allen, et al., Plasma Phys. Control. Fusion 37 (1995) A191.

[6] A.W. Leonard,et al., Rev. Sci. Instru. 66 (1995) 1201.

[7] R.A. Hulse, Nuclear Technology/Fusion 3 (1983) 259.

[8] J. Bohdansky, Nuclear Instr. Meth. B2 (1984) 587.

[9] C. García-Rosales, et al., J. Nucl.Mater. 218 (1994) 8.

[10] J. Roth, E. Vietzke, and A.A. Haasz, Suppl. Nucl. Fusion 1 (1991) 63.

[11] P. Sigmund, Phys. Rev. 184 (1969) 383.

[12] D.L. Smith, J. Nucl. Mater. 75 (1978) 20.

[13] D.L. Smith, et al., Proc. th $^{\text {th }}$ Symp. on Engineering Prob. in Fusion Research, Chicago (1981).

[14] T.E. Evans, et al., Contrib. Plasma Phys. 38 (1998) 260.

[15] D.G. Whyte, et al., these proceedings. 
16 


\section{ACKNOWLEDGMENTS}

This work is supported under U.S. Department of Energy under Contract Nos. DE-AC0389ER51114 and W-7405-ENG-48. 